\title{
Integrating preconception care for women with diabetes into primary care: a qualitative study
}

Iman Mortagy, Karina Kielmann, Stephanie E Baldeweg, Jo Modder and Mary B Pierce

\author{
ABSTRACT \\ Background \\ National guidelines emphasise the need to deliver \\ preconception care to women of childbearing age. \\ However, uptake of the services among women with \\ diabetes in the UK is low. Questions arising include \\ how best to deliver preconception care and what the \\ respective roles of primary versus secondary \\ caregivers might be.
}

Aim

To explore the perspective of GPs and secondary care health professionals on the role of GPs in delivering preconception care to women with diabetes.

\section{Design of study}

Qualitative, cross-sectional study.

\section{Setting}

A London teaching hospital and GP practices in the hospital catchment area.

\section{Method}

Semi-structured interviews with GPs and members of the preconception care team in secondary care.

Thematic analysis using the framework approach.

\section{Results}

GPs and secondary care professionals differ in their perception of the number of women with diabetes requiring preconception care and the extent to which preconception care should be integrated into GPs' roles. Health professionals agreed that GPs have a significant role to play and that delivery of preconception care is best shared between primary and secondary care. However, the lack of clear guidelines and shared protocols detailing the GP's role presents a challenge to implementing 'shared' preconception care.

\section{Conclusion}

GPs should be more effectively involved in providing preconception care to women with diabetes.

Organisational and policy developments are required to support GPs in playing a role in preconception care.

This study's findings stress the importance of providing an integrated approach to ensure continuity of care and optimal pregnancy preparation for women with diabetes.

\section{Keywords}

diabetes mellitus; general practitioners; preconception care; primary care.

\section{INTRODUCTION}

The prevalence of type 1 diabetes mellitus and type 2 diabetes mellitus in pregnancy is rising nationally and internationally, ${ }^{1-5}$ and is associated with increased risks of adverse pregnancy outcomes. . $^{2,46-12}$ Preconception care for women with diabetes is an effective means to reduce the incidence of adverse pregnancy outcomes. ${ }^{10,13-15}$ However, to date, the majority of women with diabetes enter pregnancy poorly prepared, ${ }^{1,5,6,11,13}$ and the provision of diabetes preconception care continues to be patchy across the UK. ${ }^{14}$ Historically, in the UK, diabetes care has been provided in the specialist hospital setting. The recent rise in numbers of women with diabetes becoming pregnant coincides with the restructuring of diabetes care services in the UK, which has resulted in the care of patients with type 2 diabetes being moved predominantly into primary care. ${ }^{15}$

National and international guidelines emphasise the need for health services to deliver diabetes preconception care to women of childbearing age.

I Mortagy, MSc, research fellow, Centre for Maternal and Child Enquiries, Research and Development, London. K Kielmann, $\mathrm{PhD}$, lecturer in medical anthropology, London School of Hygiene and Tropical Medicine, Department of Public Health Policy, London. SE Baldeweg, MD, FRCPI, FRCP, consultant physician in diabetes and endocrinology; J Modder, MRCOG, consultant obstetrician, University College London Hospitals NHS Foundation Trust, London. MB Pierce, MD, FRCGP, GP and clinical epidemiologist, Medical Research Council, Unit for Lifelong Health and Ageing, London.

Address for correspondence

Mrs Iman Mortagy, Centre for Maternal and Child

Enquiries, Research and Development, 188 Baker Street, London, NW1 5SD. E-mail: iman.mortagy@cmace.org.uk

Submitted: 15 January 2010; Editor's response: 16 February 2010; final acceptance: 13 May 2010.

(c)British Journal of General Practice 2010; 60: 815-821.

DOI: 10.3399/bjgp10X532594 


\section{How this fits in}

Effective preconception care is associated with improved pregnancy outcomes

for women with diabetes. This research outlines the views of GPs and

secondary care professionals involved in diabetes care on the delivery of

preconception care in London. Currently, there appears to be a lack of shared

vision about the importance of preconception care, how best to deliver the

service, and the role of GPs.

The UK Diabetes National Service Framework (NSF) suggests that preconception care should be provided jointly by the adult diabetes service and the maternity service for women wishing to become pregnant. ${ }^{16}$ In 2008, the National Institute for Health and Clinical Excellence (NICE) published a national guideline recommending that women with diabetes should be informed about the benefits of preconception glycaemic control at each contact with healthcare professionals, from adolescence onwards. ${ }^{17}$ The guideline highlighted that health services in England are currently ineffective in addressing the low uptake of preconception care, but failed to recommend an optimal delivery strategy.

A previous study suggested that primary care professionals do not rate preconception care as high in their workload priority, and offer this service opportunistically rather than routinely. ${ }^{18}$ However, there is very little evidence on what health professionals think about the division of care and their respective roles in delivering preconception care to women with diabetes. The aim of this paper is to examine the perspective of GPs and secondary care health professionals on current and envisaged roles and responsibilities of GPs in delivering preconception care to women with diabetes.

\section{METHOD}

\section{Study design}

This paper presents findings from interviews conducted as part of a larger qualitative study on diabetes mellitus in pregnancy carried out between April 2008 and January 2009. Semi-structured

\section{Box 1. Interview topics discussed with GPs and hospital} team.

Topic 1: Perceptions of the 'theory' and organisation of pre-conception care in primary and/or secondary care, and the roles of GPs and the hospital team within this model

Topic 2: The extent of GPs and the hospital teams' practical involvement in preconception care

Topic 3: The advantages and challenges of delivering and accessing 'ideal' preconception care in primary care

Topic 4: Reflections on ways of improving the delivery of preconception care interviews were conducted with 22 women, of different ethnic backgrounds, with type 1 or type 2 diabetes, who had attended one London teaching hospital. Semi-structured interviews were also conducted with health professionals associated with the hospital's multidisciplinary diabetes preconception care team $(n=7)$ as well as a sample of GPs $(n=8)$ serving the catchment area of the hospital and responsible for the hospital referrals of the women participating in the study. A qualitative approach was deemed appropriate for the study, as it allowed responders to reflect on the concept of preconception care as well as its practical delivery. This paper draws on the data collected from GPs and health professionals in secondary care.

\section{Sample and study participants}

GPs. Eighteen GPs responsible for referring all women interviewed as part of the larger qualitative study were identified from the general patient database at the hospital. Thirteen of the 18 GPs were purposively selected, from different practices, to ensure adequate representation of involvement in diabetes care (referred to in this paper as 'diabetes lead' or 'non-lead'). GPs were contacted via phone, and sent information on the study via email and post. Eight GPs agreed to be interviewed. They represented a diverse set of practices with a range of list sizes varying from 3000-16 000 patients, and a range of one to seven GP partners.

Secondary care health professionals. The seven members of the multidisciplinary preconception care team included a consultant diabetologist and a consultant obstetrician, both responsible for the diabetes maternity service, three diabetes specialist nurses, one diabetes midwife, and one dietician.

\section{Data collection}

Study participants were interviewed at a place and time that was convenient for them. Interviews were carried out by two members of the research team and lasted between 30 and 60 minutes. A semistructured interview topic guide (Box 1) was used to elicit information. All interviews were digitally recorded and transcribed verbatim.

\section{Data processing and analysis}

Interview transcripts were analysed by thematic content analysis, using the framework approach involving the following steps: (1) familiarisation with the data; (2) identifying a thematic framework; (3) indexing; (4) charting; (5) mapping and interpretation. ${ }^{19}$ MAXQDA, a software program for supporting qualitative data analysis was used to code, organise, and retrieve coded segments. The retrieved data were 
organised in charts in order to compare and contrast themes within and across groups and to find associations between themes. Descriptive themes derived from the questions were identified, as well as more analytical themes that emerged from the interpretative process of data analysis.

\section{Data validation}

Analyst triangulation and responder validation were used to ensure rigour in data interpretation. Two of the researchers met regularly throughout the duration of the data collection and analysis period. Each researcher reviewed transcripts individually to identify data segments that related to the key objectives of the study as well as to identify emergent analytical themes. Themes identified and shared by both researchers formed the basis of a coding structure that was applied to all transcripts. A responder validation meeting took place with the hospital team after initial data analysis. Due to varying schedules and GP practice workloads, it was not feasible to conduct a similar meeting with the GPs interviewed.

\section{RESULTS}

The main question addressed in the analysis was 'how do GPs and secondary care health professionals perceive and experience the division of roles in preconception care?'. Four main factors influencing the GP's role in preconception care emerged from the interviews: case load and patient profile; ambiguity of GP roles and responsibilities; missed opportunities; and integration of care.

\section{Case load and patient profile}

There were mixed views on the actual numbers of pregnant women with type 2 diabetes. For example, the GPs emphasised that only a very small number of women with diabetes per practice become pregnant. Hence, they perceived the problem of low uptake of preconception care as a problem that only affects a 'minority of minorities'. It was perceived that type 2 diabetes in most cases was associated with older patients. As one GP pointed out:

'I think part of the problem is, type 2 diabetes for a lot of people is seen as a disease of the elderly. And more of our patients are middle-aged to old people so I think the kind of, you know, diabetes, think about pregnancy, is not automatic at all, um, and the numbers are small, small, small.' (GP2, diabetes lead)

On the other hand, the hospital team reported seeing many more pregnant women with type 2 diabetes with uncontrolled levels of blood glucose, to whom they had no access prior to pregnancy. They highlighted that the GP's role as a key member of the multidisciplinary professional team was crucial, yet missing. Hospital team members emphasised that the role of the GP was particularly relevant to young women with type 2 diabetes:

'I keep repeating that, you know, is it possible for the GPs that see all the type two young mothers, you know, to rub it in more often that, you know, please do plan and come for preconception before you even think of a pregnancy. But that's where it falls down.' (diabetes specialist nurse 2)

\section{Ambiguity of GP roles and responsibilities in preconception care}

The GPs in the study sample expressed differing opinions regarding their role in preconception care. The divergent opinions in this group may stem from their varying degree of involvement in diabetes care. For example, GPs with a special interest in diabetes perceive themselves as highly involved and responsible for the care of the woman before pregnancy and hence capable of providing some strands of preconception care as expressed by a diabetes lead:

'If they were young and fertile, l'd make sure their drugs were ... safer if they got pregnant and I would get their control as good as it was and I'd emphasise they must come and see us as soon as they're pregnant.' (GP1, diabetes lead)

On the other hand, GPs with no special interest in diabetes were less clear about their involvement in the pre-pregnancy care of women with diabetes. A GP reflects:

I've never really thought about this topic before but ... I would say it's definitely in primary care, we have to be aware, we have to highlight these cases where we probably hadn't been doing.' (GP5, non-diabetes lead)

The lack of clarity in responsibilities was further attributed to the targets set up by the commissioning bodies regarded as 'mixed messages' and 'conflicting incentives' by some GPs:

'The government has set up conflicting incentives. On the one hand you must identify all disease groups and all risks, and be judged upon how well you do that. On the other hand if you refer patients to hospital, you're going to be financially penalised.' (GP7, diabetes lead)

Similarly, there was no consensus among 
members of the multidisciplinary preconception care hospital team regarding what GPs' involvement should be. Expectations of their role ranged from awareness raising only, to the possibility of GPs providing all the required tests and check-ups. There was some scepticism regarding GPs' interest and capability to take on preconception care fully:

'I would be very happy for GPs to deliver preconception care and then to tap into our system, into our team, if I thought they could ... if I thought they were delivering the goods the way that we do it ... The problem is that some GPs have a lot of interest in diabetes and are probably already doing it and doing it very well, and there are some GPs who have no interest at all and their general level of diabetes care is very poor.' (hospital consultant)

This was in contrast to another hospital professional who believed that more aspects of preconception care could be delivered in primary care:

'I think diabetes has started changing, and a lot of it is happening, even the specialist stuff is now happening in the community. So if there was a specialist community clinic ... in a GP practice, or whatever, then that would work well, because it would be seeing them close to home.' (dietician)

There was consensus among all interviewees that GPs are best suited to reach high-risk women. They should therefore play a proactive role in informing and educating women about preconception care issues:

'I don't think it matters who gives the message as long as we're all giving the same, consistent message, fantastic, GPs, practice nurses, health visitors, the lot, community midwives, everyone, we should all be in it together actually. I don't think it should just be us.' (diabetes specialist nurse 1)

'We should be really telling all women of, you know, of a certain age, that they do need to plan their pregnancies really if they're diabetic, yeah. So certainly I would absolutely agree with that, that the role is in primary care for us to educate them before it's too late really.' (GP5, nondiabetes lead)

\section{Missed opportunities}

Disparity in diabetes care practice among GPs within the same surgery, as well as questions around the division of responsibility between primary and secondary care, amounted to a source of tension. In some instances, the lack of a clear division of labour led to missed opportunities for patient care, as illustrated in the story related by one GP:

'Although we tried to engage her [the patient] here as well as her seeing the hospital ... we then didn't make her come in and see us. You know, she knew we were available. So, because we weren't doing the organised care, we won't have gone through the template in a "we must complete every line" way. Also, she was under a different doctor at the surgery and because she wasn't seeing me in diabetic clinic ... it didn't seem like important enough to change her regular doctor, because the hospital was looking after her diabetes.' (GP1, diabetes lead)

The hospital team agreed that the rise in numbers of pregnant women with type 2 diabetes in addition to the lack of a defined GP role in preconception care may lead to many missed referral opportunities. The hospital team underlined the scarcity of preconception care referrals received from primary care compared to those received from within the hospital:

'A key professional is missing. In fact [the GP] he or she is the most important professional, because they are bringing the woman to this preconception care [clinic], so, that's gone, that's missing.' (hospital consultant)

'Well, I think they're [assisted conception unit in hospital] very good, because I get some referrals from them, whereas, I don't get any GP referrals for preconception care. I cannot remember if we have ever had one.' (hospital consultant)

The secondary care team pointed out the need for a joint in-house strategy as well as a clear shared vision with primary care, with GPs being active members of the team providing the preconception care service. The lack of referrals was linked by both groups of professionals to an absence of clear guidelines on how to provide preconception care and when to make referrals:

'If they're not pregnant when do you make the referral without hugely increasing the workload of the clinic at hospital? Will they [hospital team] be able to cope, if you're referring 10 women who are trying to get pregnant but none of them are pregnant, it's a huge increase in workload.' (GP5, non-diabetes lead) 
GPs were unsure in some cases regarding what was offered to women in preconception care clinics in secondary care. Unsurprisingly, only two GPs could name hospitals with preconception care clinics:

'If you've proven good diabetic control and you're checking stuff before you stop the contraception and you're taking folic acid, what extra do they do for the diabetic women in these clinics? I don't know.' (GP8, diabetes lead)

\section{Integration of care}

GPs envisaged preconception care as a 'joint responsibility' between primary and secondary care, with all relevant checks and advice offered to women in primary care, while specialist issues such as insulin adjustment are led by secondary care:

'I think it is our role to do the preconception check, the rubella, do the contraception, start the folic acid.' (GP1, diabetes lead)

'That [preconception care] is a situation where you might do a bit of collaboration and mix and match ... I might say why don't you [the patient] go and have a one-off appointment, talk about what are issues for preconception care, maybe ... you've got to get some target sugars ... or you have got to convert to insulin, if you're not using it and then let us [primary and secondary care] share the care.' (GP4, non-diabetes lead)

Continuity of care in both primary and secondary care was highlighted as an important aspect of an effective preconception care service. GPs emphasised that women should be offered an integrated service that ensures continuity from preconception through maternity in secondary care. This service would ideally be offered as part of the general diabetes care:

'I think you don't want to be having your diabetic care separate from your antenatal care, I think they need to be joined together. And I'm not sure you need your preconceptual care separate from your diabetic care.' (GP4, non-diabetes lead)

'You're not dealing with diabetes you're dealing with a woman with diabetes. And if you actually separate that woman off from the diabetes, there's a mistake. So I think that actually it should be part of general diabetic care.' (GP6, non-diabetes lead)

The GPs suggested that locally agreed practice protocols are required to streamline preconception care into GPs' workload. They argued that preconception care should be better integrated into their daily work: firstly, preconception care could be added to the diabetes template and pop-up reminders or alert messages could be used:

'People often go by what's on the templates and, you know, that's not a part of it. So, I think if you're rushing and you're filling in templates, it's not on it, it might be an idea to put that in ...' (GP8, diabetes lead)

'Frankly [in] general practice, you're under such pressure in terms of time in a general consultation, you know, you might have 10 minutes, it's um, unless there is a system that alerts you to um, to consider it, it's unlikely to get into, you know, you're just dealing with now.' (GP7, diabetes lead)

Secondly, GPs argued that preconception care should be offered routinely, to all women in their childbearing years - rather than opportunistically to those who express the wish to start a family. It was suggested that it may be helpful to introduce a routine preconception care referral to secondary care for all newly diagnosed women. This shift would reduce the possibility of some women being missed:

'I actually think rather than talking about preconception care, you talk about the ... care of the potentially pregnant rather than preconception, because preconception care suggests it is an active decision to actually conceive, and therefore you'll miss a lot of people because people won't be ready for preconception care advice.' (GP6, non-diabetes lead)

'So at time of diagnosis you refer everybody. Um, and that would actually be, I think, the best, yeah, that would be brilliant ... And you could have a box on the computer where you tick it if they've, they've been referred ... So then at least you know they've had that information once.' (GP3, diabetes lead)

Thirdly, several GPs and hospital team members emphasised the need to raise awareness among health professionals through ongoing education and training:

'It's highlighting to the GPs to send most of the type ones or type twos, you know, the young mothers ... just keep them aware to ring us for an 
appointment for preconception clinic.' (diabetes specialist nurse 2)

'If it was raised in our awareness, then as GPs we could just say in passing, you know, you are, you do know the story about if you were to get pregnant. And I suspect we don't, I suspect we also expect that they will be seen largely in secondary care.' (GP4, non-diabetes lead)

Lastly, GPs highlighted the need to be supported by evidence-based information on preconception care benefits, as well as having access to patient information leaflets that could be handed out when offering the service:

'Well the first thing is ... [a] clear evidence ... base of the advantages of preconception. In other words, basically being quite certain of the endeavour you're about to start, has proven benefit ... Second thing is ... an easy way of communicating that to primary care staff, so they've got ... access to that information ... to pass to patients, nice summary, preferably an insert in their patient-held record, which should be very easy to do.' (GP7, diabetes lead)

\section{DISCUSSION}

\section{Summary of main findings}

GPs and secondary care health professionals differ in their perception of the number of women requiring preconception care. On the one hand, GPs stress the very low number of women with diabetes likely to become pregnant in their practices. On the other hand, secondary care is dealing with many more pregnant women with diabetes who enter pregnancy poorly prepared. Both groups of professionals agree that GPs have an important role to play in providing preconception care, despite differences in opinion regarding what exactly this role should be. Perceived mixed messages from the commissioning bodies and a lack of clear local preconception care policies, guidelines, and referral pathways contribute to ambiguity in the GP's role. All health professionals interviewed agreed that this lack of clarity may lead to missed opportunities for patient care.

\section{Strengths and limitations of the study}

As far as the researchers are aware, this is the first study to elicit in-depth perspectives of GPs and secondary care health professionals in the UK regarding the delivery of preconception care for women with diabetes.

Through a focus on one hospital and its catchment area, the research aimed to provide a rich case study. While one can be confident that the findings are indicative of some of the issues and tensions in integrating preconception care into primary and secondary diabetes care, they may not present perspectives of all clinical disciplines that may be involved in this area. Further research is needed to explore the perspectives of health professionals such as primary care nurses, family planning practitioners, community-based midwives, and junior doctors involved in delivering general diabetes care.

\section{Comparison with existing literature}

This study highlights a number of challenges to delivering preconception care in primary care, including the lack of clear policies and guidelines on delivering preconception care, the need for more effective communication between primary and secondary care, variation in diabetes expertise among GPs, and their time constraints. ${ }^{18,20}$ Moreover, both GPs and secondary care health professionals may lack awareness of the need to give preconception care to every woman with diabetes of reproductive age. ${ }^{11,21}$

The discrepancy in GPs' and secondary healthcare professionals' views regarding the magnitude of the problem may be explained by the current low case load per GP practice and a prevalent misperception that type 2 diabetes is more benign and only affects older women. ${ }^{5}$ However, the changing patient profile and rising numbers of pregnant women with type 2 diabetes underline the urgency of integrating preconception care into primary care systems. To this end, there is a need for better integration and division of resources between primary and secondary care. Primary care trusts have an important role to play in resolving uncertainties over how preconception care should be delivered and in supporting primary care teams with sufficient resources to deliver this service effectively. ${ }^{18}$ Existing structures could be used, for example, by integrating preconception care into structured diabetes education programmes..$^{15,22}$

Preconception care for women with diabetes comprises several elements that may need to be delivered by different services. GPs who provide routine health care to women with diabetes, especially type 2 diabetes, are in a unique position to educate women about preconception care. The suggestion by some GPs that preconception care should be offered routinely is supported by other study findings..$^{5,17}$ Some authors specify the need for diabetes preconception care to start from puberty, ${ }^{5,21}$ while others suggest that all health professionals should consider every visit with women of childbearing age to be a preconception visit. ${ }^{17,23}$ 


\section{Implications for clinical practice and future research}

The study findings point to the need for a number of organisational and policy developments to support GPs in a proactive diabetes preconception care role. Preconception care needs to be integrated into primary care, both as a concept and as clinical practice; however, it is best delivered collaboratively between primary and secondary care, with each sector utilising its strengths to provide specific aspects of the care. Therefore, it is pertinent that commissioners establish which aspects of preconception care can be handled effectively and safely in primary care.

The perception that type 2 diabetes is a disease of older people needs to be addressed, and healthcare professionals need to be trained to view preconception counselling as ongoing information to be given to every woman of reproductive age diagnosed with diabetes from the onset of her condition.

There is an urgent need for locally agreed practice protocols that clarify the context and timing of preconception care referrals to secondary care. Practical developments to current systems, such as automatic software reminders and leaflets with preconception care information, may support GPs in delivering diabetes preconception care within their time constraints.

This study highlights the disparity in diabetes care practice among GPs within the same surgery. Future research should take this into consideration and include a diversity of other GP characteristics such as sex and age. There is a need to examine alternative ways and implications of integrating preconception care into primary care systems. Additionally, case studies that highlight examples of successful primary and secondary care interaction could be used as models of best practice.

\section{Funding body}

This research was supported by a grant from Novo Nordisk Limited, reference number 07/H0715/50. The authors' work was independent from the funders.

\section{Ethics committee}

Ethical approval was obtained from The Joint UCL/UCLH Committees on the Ethics of Human Research Committee Alpha. R\&D approval was obtained from the Joint UCLH/UCL Biomedical Research (R\&D) Unit and from North Central London Research Consortium responsible for Islington and Camden PCTs and Haringey TPCT. Reference number 08/0058 (Joint UCLH/UCL Biomedical Research Unit).

\section{Competing interests}

Two of the hospital team members interviewed in the study (Jo Modder and Stephanie Baldeweg) are contributing authors. However, they were not involved in the recruitment process, design of the health professional interview guides, or data analysis and interpretation.

\section{Acknowledgements}

We would like to thank all primary and secondary care health professionals who agreed to be interviewed as part of this study. We would also like to thank Heather Hughes for administrative support and Rosie Houston for comments on an earlier version of this manuscript.

\section{Discuss this article}

Contribute and read comments about this article on the Discussion Forum: http://www.rcgp.org.uk/bjgp-discuss

\section{REFERENCES}

1. Bell R, Bailey K, Cresswell T, et al. Trends in prevalence and outcomes of pregnancy in women with pre-existing type I and type II diabetes. BJOG 2008; 115(4): 445-452.

2. Clausen T, Mathiesen E, Ekbom P, et al. Poor pregnancy outcome in women with type 2 diabetes. Diabetes Care 2005; 28(2):323-328.

3. Coulthard T, Hawthorne G. Type 2 diabetes in pregnancy: more to come? Pract Diabet Int 2008; 25(9):359-361..

4. Dunne F, Brydon P, Smith K, Gee H. Pregnancy in women with type 2 diabetes: 12 years outcome data 1990-2002. Diabet Med 2003; 20(9): 734-738.

5. Slocum JM. Preconception counselling and type 2 diabetes. Diabetes Spectrum 2007; 20(2): 117-123.

6. CEMACH. Pregnancy in women with type 1 and type 2 diabetes in 2002-03, England, Wales and Northern Ireland. London: CEMACH; 2005.

7. Hughes R, Rowan J. Perinatal outcomes and macrosomia in a multiethnic population of women with type 2 diabetes. Aust N Z J Obstet Gynaecol 2006; 46(6): 552-555.

8. Keely E. Type 2 diabetes in pregnancy: importance of optimized care before, during and after pregnancy. Obstet Med 2008; 1: 72-77.

9. Persson M, Norman M, Hanson U. Obstetric and perinatal outcomes in type 1 diabetic pregnancies: a large, population-based study. Diabetes Care 2009; 32(11): 2005-2009.

10. Ray JG, O'Brien TE, Chan WS. Preconception care and the risk of congenital anomalies in the offspring of women with diabetes mellitus: a meta-analysis. QJM 2001; 94(8): 435-444.

11. Roland JM, Murphy HR, Ball V, et al. The pregnancies of women with type 2 diabetes: poor outcomes but opportunities for improvement. Diabet Med 2005; 22(12): 1774-1777.

12. Temple R, Aldridge V, Murphy H. Pre-pregnancy care and pregnancy outcomes in women with type 1 diabetes. Diabetes Care 2006; 29(8): 1744-1749.

13. Cartwright A, Wallymahmed M, Macfarlane I, Casson I. What do women with diabetes know about pregnancy and contraception? Practical Diabetes International 2009; 26(6): 238-242.

14. CEMACH. Diabetes in pregnancy: are we providing the best care? Findings of a national enquiry. London: CEMACH, February 2007.

15. McDowell JRS, McPhail K, Halyburton G, et al. Perceptions of a service redesign by adults living with type 2 diabetes. J Adv Nurs 2009; 65(7): 1432-1441.

16. Department of Health. National service framework for diabetes: delivery strategy. London: DOH, 2003.

17. NICE. Diabetes in pregnancy: management of diabetes and its complications from preconception to the postnatal period. London: NICE, 2008.

18. Heyes T, Long S, Mathers N. Practice and beliefs of primary care workers. Fam Pract 2004; 21(1): 22-27.

19. Ritchie J, Lewis J (eds). Qualitative research practice: A guide for social science students and researchers. London: SAGE publications, 2003.

20. Owens MD, Kieffer EC, Chowdhury FM. Preconception care and women with or at risk for diabetes: implications for community intervention. Matern Child Health J 2006; 10(5 Suppl): S137-S141.

21. Curtis M, Abelman S, Schulkin J, et al. Do we practice what we preach? A review of actual clinical practice with regards to preconception care guidelines. Matern Child Health J 2006; 10(5 Suppl): S53-S58.

22. Lawton J, Parry O, Peel E, Douglas M. Diabetes service provision: a qualitative study of newly diagnosed type 2 diabetes patients' experiences and views. Diabet Med 2005; 22(9): 1246-1251.

23. Holing E. Preconception care of women with diabetes: the unrevealed obstacles. J Matern Fetal Med 2000; 9(1): 10-13. 